\title{
The European Society of Endocrine Surgeons perspective of thyroid cancer surgery: an evidence-based approach
}

\author{
Kerstin Lorenz • Bruno Niederle • Thomas Steinmüller • \\ Henning Dralle
}

Received: 22 December 2013 / Accepted: 25 December 2013

(C) Springer-Verlag Berlin Heidelberg 2014

During the last two decades, thyroid cancer surgery has dramatically changed due to the rising incidence of small papillary thyroid cancers, the identification of RET proto-oncogene mutations causing hereditary thyroid cancer, the introduction of minimally invasive procedures including scarless neck surgery, and the acknowledgement of BRAF mutation as a promising diagnostic element for presurgical diagnosis of papillary thyroid cancer. The European Society of Endocrine Surgeons (ESES) Workshop 2013 on "Thyroid Cancer Surgery" in Berlin was aimed to summarize and discuss the aforementioned recent developments concerning their conceptual consequences for the diagnosis and surgical approach to thyroid cancer. The design of the workshop was basically evidence based. The presentations of major sessions were thoroughly prepared ahead to the meeting by a group of responsible authors each. Final manuscripts published in this issue of the Langenbeck's Archives of Surgery (1$6,9-11)$ are representing the conclusion of pre-workshop electronic versions and discussions by the ESES members' assembly throughout the sessions of the congress.

\section{Timing and extent of surgery for gene carriers of hereditary $\mathrm{C}$-cell disease}

Based on the current evidence of hereditary medullary thyroid cancer (MTC), the assembly agreed that tailoring

K. Lorenz $(\bowtie) \cdot H$. Dralle

Department of General, Visceral and Vascular Surgery,

University of Halle-Wittenberg,

Ernst-Grube-Strasse 40, 06097 Halle (Saale), Germany

e-mail: kerstin.lorenz@medizin.uni-halle.de

B. Niederle

Department of General Surgery, University of Vienna,

Währinger Gürtel 18-20, 1090 Vienna, Austria

T. Steinmüller

Department of General-, and Visceral Surgery, DRK-Clinics

Westend, Spandauer Damm 130, 14050 Berlin, Germany surgery according to the genotype of RET proto-oncogene mutation, age, and calcitonin level appears superior to fixed risks groups based on the genotype and age only [1]. For a true prophylactic thyroidectomy in asymptomatic gene carriers of hereditary $\mathrm{C}$-cell disease, the basal calcitonin level is of utmost importance. Most recent studies have convincingly shown that C-cell-derived MTC is confined to the thyroid gland unless basal calcitonin level exceeds the upper normal range.

In order to individualize the optimum time for prophylactic thyroidectomy and to minimize surgical morbidity, in particular permanent postoperative hypoparathyroidism, asymptomatic gene carriers of hereditary C-cell disease should be prepared for parathyroid-preserving prophylactic thyroidectomy independent of age and genotype but based on basal calcitonin levels. The best time for curative prophylactic thyroidectomy is when basal calcitonin levels are approaching the upper normal range. Due to the rarity of the disease and the surgical challenges of performing thyroidectomy in the pediatric age, only highly specialized thyroid surgeons should perform prophylactic thyroidectomies in children.

\section{Multifocal papillary thyroid cancer}

Multifocal papillary thyroid cancer (PTC) is a unique subtype of PTC concerning its controversial pathogenesis from intrathyroidal spread of primarily unifocal PTC or multifocal synchronous de novo lesions of PTC [2]. Apart from underlying risk factors like previous external irradiation of the neck or familial non-medullary thyroid cancer, there is no general consensus regarding the frequency of regional lymph node metastases of multifocal PTC compared to unifocal PTC of the same tumor size. The role for prophylactic central node dissection (CND) in multifocal PTC therefore is controversially discussed. 
Based on the current literature, the assembly agreed that multifocal PTC should be surgically treated by total instead of less than total thyroidectomy. This may require completion thyroidectomy in cases diagnosed only by final histopathology. When multifocal PTC has been diagnosed preoperatively or intraoperatively, prophylactic central node dissection should be considered also for micro-PTC. However, there is no sufficient evidence for CND as a completion procedure for cN0 PTC when total thyroidectomy already has been performed. Depending on the postoperative thyroglobulin levels, radioiodine ablation may be an option for diagnosis of metastatic disease and follow-up.

\section{Minimally invasive follicular thyroid cancer}

To be separated histopathologically and clinically from widely invasive follicular thyroid cancer (WIFTC), minimally invasive follicular thyroid cancer (MIFTC) is characterized by minimal capsular invasion with or without angioinvasion. Most surgeries are performed for follicular neoplasia, and the diagnosis relies on final histopathology including several sections throughout the tumor confirming the diagnosis. Several studies have conclusively shown that MIFTC with capsular invasion only, independent from the tumor size does not have any metastatic risk, thus not requiring completion thyroidectomy with radioiodine treatment after less than total thyroidectomy [3].

In contrast to minimal capsular invasion, angioinvasion has been proven to be associated with distant metastases. The risk for metastases may be correlated to the number of angioinvasions; however, there are no studies corroborating a quantitative relationship between both. In the case of less than total thyroidectomy with postoperative histopathological diagnosis of angioinvasive MIFTC, completion thyroidectomy with radioiodine ablation is strongly recommended.

Because lymph node metastases in MIFTC are completely uncommon, prophylactic CND as part of the primary or completing procedure therefore was not recommended by the assembly.

\section{Prophylactic central nodal dissection in papillary thyroid cancer}

Prophylactic CND in cN0 PTC has been intensively studied during the last two decades. Due to the overall low frequency of the disease and the favorable outcome, prospective studies concerning the benefit of prophylactic CND were not feasible up to now. The concept of prophylactic CND in cN0 PTC therefore hinges basically on risk-benefit evaluations concerning surgical morbidity on the one side, and recurrence and survival rates on the other side.

Data in four out of five recent meta-analyses have confirmed higher complication rates at least for transient hypoparathyroidism after CND [4]. Due to the limited power of the studies, there was only a trend to increased permanent hypoparathyroidism rates after CND, but this was not statistically significant. The rates of post-CND transient and permanent recurrent laryngeal nerve palsies were not increased as shown by all published series.

In contrast to the increased surgical morbidity after prophylactic CND even in experienced hands, there is some evidence that thyroglobulin levels may be lowered by prophylactic CND along with better staging and removal of incidental micrometastases. Whether slightly increased thyroglobulin levels, however, are representing relevant outcome-related tumor burden has not been conclusively shown.

Based on the current literature, the assembly finally agreed that there is no indication for routine CND in cN0M0 PTC unless there are clinical risk factors like multifocality or extrathyroidal invasion.

\section{BRAF mutation in papillary thyroid cancer}

The presence of BRAF mutations has been shown to be a characteristic feature of PTC in contrast to FTC and may correlate to a more aggressive course of the disease compared to BRAF-negative PTC. BRAF mutational analysis now is routinely available by fine needle aspiration cytology [5]. Concerning the surgical consequences of preoperative diagnosis of BRAF-positive PTC, most of the participants agreed that there is a need for total thyroidectomy; however, there was no unanimously preference for prophylactic CND in cN0 BRAF-positive PTC.

\section{Classification systems of locoregional lymph nodes in thyroid cancer}

Actually, there are four classification systems for locoregional lymph nodes in thyroid cancer (USA, Japan, UICC, and Germany) with some pros and cons in each concerning the definition of locoregional versus systemic disease, the landmarks of subgroups, and last but not least the extent of compartment dissection for papillary and medullary thyroid cancer [6]. The German Association of Endocrine Surgeons adapted the compartment classification [7] due to its simplicity, the clearly defined landmarks which include also the infrabrachiocephalic upper mediastinum, and the separate definition of neck and mediastinal compartments for the right and left side. 


\section{Robotic thyroidectomy}

Modern patient demands concern improved cosmesis as well as reduction of complications. Thyroid surgery minimized incisional length and pain and involved video-assisted and fully endoscopic techniques. The quest for a scarless neck surgery entailed anterior chest, breast, and axillary approaches, that Chung pioneered in a gasless robot assisted technique. The Asian-dominated propagation of robotic thyroidectomy in the European or American setting is confronted with major cultural and constitutional differences: The ambition to scarless neck in Europeans, and moreover predominantly female patients, falls behind concerns against areolar breast incisions.

The majority of European and American patients differ from the extraordinarily slender, lean, and smaller Asians, eventually necessitating further and longer technical equipment and modification of the technique. Concerns furthermore involve new and severe complications not known in open thyroid surgery like brachial plexus neuropathy and tracheal injury, as well as quality control regarding recurrent laryngeal nerve palsy and hypoparathyroidism, moreover adequacy of oncological outcome in thyroid cancer. While longer procedural time is accepted in the learning curve, immense cost expenditure and considerate complication rates are highly controversial [8]. Even though the procedure and cosmetic outcome are elegant, propagation of robotic thyroidectomy in Europe struggles with expenditure in costs, time, learning curve, constitution, and missing oncological outcome and follow-up data.

\section{Sentinel node biopsy in papillary thyroid cancer}

Sentinel node biopsy (SNB) in papillary thyroid cancer (PTC) using blue dye and/or radioisotope aims at selecting patients with occult node metastasis for therapeutic lymphadenectomy and sparing the remaining overtreatment associated with increased rates of hypoparathyroidism and recurrent laryngeal nerve palsy. Despite general feasibility, SNB in thyroid cancer is restricted by practical limitations: Only a minority of patients may potentially benefit, radioisotope injection requires logistic efforts, and the necessary node exposure inhibits the predicament of disrupting lymphatic drainage. Moreover, PTC with occult node metastasis is rarely evidenced preoperatively, and SNB only applicable in primary surgery.

In a retrospective cohort study, evaluation of SNB proved ineffective in costs and reduction of morbidity in avoiding central node dissection in patients with negative sentinel nodes, even if ideal conditions and results were assumed [9]. The specific properties of thyroid cancer preclude recognizable benefit of SNB in a relevant number of patients in regard to reduction of morbidity at considerate cost amendment.

\section{Classification of aerodigestive tract invasion by thyroid cancer}

Only less than $5 \%$ of thyroid cancers are invading the aerodigestive tract. Both the extent of horizontal invasion, namely at the larynx and trachea wall, and the site and length of longitudinal invasion are determinants of the surgical strategy. While shaving procedures are reserved for palliation and non-transmural invading cancers, window and sleeve resections are the optimum treatment for thyroid cancers with transmural invasion [10]. Due to the nonsignificant survival differences in favor of shaving procedures compared to window and sleeve resections at least for thyroid cancers with limited trachea invasion tumor biology, age, general condition of the patient, and surgical risk have to be taken into consideration concerning the best choice of surgical treatment.

\section{Intraoperative nerve monitoring in thyroid cancer surgery}

The main gain of intraoperative nerve monitoring (IONM) compared to visual nerve identification alone exhibits neural functional ascertainment. Specific conditions of thyroid cancer surgery emphasize merits of IONM: faster identification of the recurrent laryngeal nerve (RLN) in scarred tissue, mapping the neural course, and minimizing unnecessary dissection. Functional neural prognostication permits staged surgery in case of loss of signal associated with postoperative vocal cord dysfunction. In patients with intact vocal cord mobility preoperatively, preservation of the RLN exhibiting tumor infiltration may be balanced in favor of time with voice containment by adjunctive oncological concepts [11].

Despite considerable utility of IONM, conflicts regarding unsatisfactory positive predictive power, reliable definitions of adequate basal stimulation values, and loss of signal remain to be resolved. These aspects all impact the concept of staged thyroidectomy in the event of IONM changes in quest to prevent bilateral vocal cord palsy, even more so in thyroid cancer. However, the recent propagation of continuous IONM (CIONM) may prospectively assist in answering some of the questions by promoting recognition of incipient nerve injury with option to modify surgical procedures, and overcome the limits of intermittent IONM where possible neural damage in between vagal stimulation remains undetected. Thus, functional assessment and provision of neural prognosis by CIONM are expected to further affect surgical strategy intraoperatively with tribute to effecting staged thyroidectomy and 
decision of nerve preservation or sacrifice in thyroid cancer surgery.

\section{Treatment and follow-up of low risk differentiated thyroid cancer}

A majority of thyroid cancer patients reveal low risk differentiated thyroid cancer (DTC). The low risk of recurrence and even lower risks for death demand improvement of preoperative selection criteria for safely decreasing the extent of surgical procedure, adjunct treatment, and follow-up measures. Established concepts for DTC rely on retrospective study data, and conduction of prospective multicentric randomized studies was dismissed as impossible for rarity and costs. Despite this, following industrially sponsored studies in orphan disease like tyrosine inhibitors in refractory thyroid cancer, multicentric design enabled sufficient numbers in rare disease (DECISION and SELECT trials). The realization of these randomized studies conveyed approval of new concepts like rhTSH in follow-up and ablation in low risk DTC. Recently, ultrasensitive thyroglobulin assessment and obviation of radioiodine ablation are investigated.

Distributing microcarcinomas to only undergo follow-up and DTC requiring treatment is improved by cytologic classification and BRAF on fine needle aspiration (ESTIMABL and ESTIMABL2). Adaption of easier and cost-effective follow-up with sensitive $\mathrm{Tg}$ in prospective analysis further assists quality of life (THYRDIAG). Thus, the challenges in the treatment and follow-up of low risk DTC are still addressed in larger multicentric studies aiming at evidence for stratification and tailoring conservative, investigative, and therapeutic measures of treatment and follow-up adequately. Even though the need for industrial involvement in realization of these studies of rare disease appears indispensable, enormous challenge lies in the need to identify and counterbalance industrial interests and study bias.

\section{Conclusion}

In conclusion, the 2013 European Society of Endocrine Surgeons' evidence-based workshop has shown that thyroid cancer surgery during the last two decades is moving on by new diagnostic methods and refinements of surgical techniques from a single standard procedure for all types of thyroid cancer to a more and more type- and stage-oriented approach. There is increasing evidence that low risk thyroid cancers like clinically node-negative papillary microcarcinoma and minimally non-angioinvasive follicular thyroid cancer can be treated less aggressively than in the past without oncologic disadvantage but less surgical morbidity. However, although the feasibility of prospective randomized studies has been questioned [12], the endocrine surgical community should undertake all efforts to initiate a prospective randomized study at least concerning prophylactic CND for papillary thyroid cancer, the most frequent type of thyroid cancer worldwide. New multidirectional molecular tests will assist in optimizing the surgical strategy in order to avoid both undertreatment and overtreatment of thyroid cancers.

\section{Conflicts of interest None.}

\section{References}

1. Niederle B, Sebag F, Brauckhoff M (2014) Timing and extent of thyroid surgery for gene carriers of hereditary C cell disease - a consensus statement of the European Society of Endocrine Surgeons (ESES). Langenbeck's, Arch Surg 399: 185-197

2. Iacobone M, Jansson S, Barczynski M (2014) Multifocal papillary thyroid carcinoma - a consensus report of the European Society of Endocrine Surgeons (ESES). Langenbeck's, Arch Surg 399: $141-154$

3. Dionigi G, Kraimps JL, Schmid KW, Hermann M (2014) Minimally invasive follicular thyroid cancer (MIFTC) - a consensus report of the European Society of Endocrine Surgeons (ESES). Langenbeck's, Arch Surg 399:165-184

4. Sancho JJ, Lennard TW, Paunovic I, Triponez F (2014) Prophylactic central neck dissection in papillary thyroid cancer: a consensus report of the European Society of Endocrine Surgeons (ESES). Langenbeck's, Arch Surg 399:155-163

5. Miccoli P, Basolo F (2014) BRAF mutation status in papillary thyroid cancer: significance for surgical strategy. Langenbeck's, Arch Surg 399:225-228

6. Musholt T (2014) Classification of locoregional lymph nodes in medullary and papillary thyroid cancer. Langenbeck's, Arch Surg 399:217-223

7. Dralle H, Musholt TJ, Schabram J, Steinmüller T, Frilling A, Simon D, Goretzki PE, Niederle B, Scheuba C, Clerici T, Hermann M, Kußmann J, Lorenz K, Nies C, Schabram P, Trupka A, Zielke A, Karges W, Luster M, Schmid KW, Vordermark D, Schmoll HJ, Mühlenberg R, Schober O, Rimmele H, Machens A for the German Societies of General and Visceral Surgery, Endocrinology, Nuclear Medicine, Pathology, Radiooncology, Oncological Hematology, German Thyroid Cancer Patient Support Organization (2013) German Association of Endocrine Surgeons practice guideline for the surgical management of malignant thyroid tumors. Langenbeck's Arch Surg 398:347-375

8. Dralle H (2013) Robot-assisted transaxillary thyroid surgery: as safe as conventional-access thyroid surgery? Eur Thyroid J 2: $71-75$

9. Balasubramanian S, Brignall J, Lin HY, Stephenson T, Wadsley J, Harrison B, Craig W, Smart L, Krukowski (2014) Sentinel node biopsy in papillary thyroid cancer - what is the potential? Langenbeck's, Arch Surg 399:245-251

10. Brauckhoff M (2014) Classification of aerodigestive tract invasion from thyroid cancer. Langenbeck's, Arch Surg 399: 209-216

11. Randolph G, Kamani D (2014) Intraoperative neural monitoring in thyroid cancer surgery. Langenbeck's, Arch Surg 399: 199-207 
12. Carling T, Carty SE, Ciarleglio MM, Cooper DS, Doherty GM, Kim LT, Kloos RT, Mazzaferri EL, Peduzzi PN, Roman SA, Sippel RS, Sosa JA, Stack BC, Steward DL, Tufano RP, Tuttle RM, Udelsman $\mathrm{R}$, American Thyroid Association Surgical Affairs Committee
(2012) American Thyroid Association design and feasibility of a prospective randomized controlled trial of prophylactic central lymph node dissection for papillary thyroid carcinoma. Thyroid 22:237-244 